topography appeared to be a feature of these deposits, and in a number of cases a levelling of the nodules was the only indication of wear.

The two neutral organic solutions investigated produced widely different wear characteristics from which no conclusion could be drawn.

With dissimilar deposits it was found in general that the results were intermediate to those of the individual deposits when tested in similar combinations, but if the inferior gold was used on the male surface and the acid cyanide gold on the female, the results were significantly worse than with the reverse combination.

The mechanism of wear for the sulphite golds and the inferior organic gold involved the apparent welding together of opposing faces, leading to material being torn from the deposit and either transferred to the opposite contact or left as debris at the ends and edges of the wear tracks. The example reproduced here in Fig. 6 shows the accumulation of a number of platelets at the end of a track.

Wear of all the acid golds and the better of the organically hardened neutral golds was in the nature of a light burnishing or scoring action, but during the course of the scanning electron microscope studies Souter considers that he found features in the wear tracks of the acid gold deposits which suggested a degree of lubrication from co-deposited polymer. Such organic films are visible in Fig. 7, taken from the paper. This type of surface film phenomenon, involving dark coloured reservoirs of polymer, could not be observed as readily, if at all, on sulphite deposits.
The conclusions put forward by Souter are that any gold deposit of purity greater than 99 per cent will cold pressure weld in the conditions in a separable connector, as evidenced by the behaviour of pure gold, silver hardened alkaline golds, sulphite golds and possibly certain neutral golds with organic additives. The excellent performance of acid cyanide deposits he ascribes to the co-deposition of polymers catalysed by the cobalt or nickel additives, these forming reservoirs of lubricant that prevent or minimise adhesion.

This view remains to be reconciled with the conclusions arrived at by Antler in the first paper summarised in this review. It is the latter's contention that the durability of gold is little, if at all, related to any surface lubrication effect of polymer, but that it is conceivable that polymer may well improve the mechanical properties of a deposit and so indirectly contribute to durability by enhancing the capacity of the deposit to respond to traces of adventitious lubricants. This view, he considers, is consistent with the observed improvement in the durability of thin gold deposits when using hard rather than soft underplates or substrates.

L. B. $\mathrm{H}$.

\section{References}

1 M. Antler, A.S.L.E.Trans., 1968, 11, (3), 248

2 A. J. Solomon and M. Antler, Plating, 1970, 57, 812

3 Gold Bulletin, 1972, 5, (4), 85

4 G. B. Munier, Plating, 1969, 56, 1151

5 L. Holt and J. Stanger, Trans. Inst. Met. Fin., 1972, $50,(1), 24$

6 M. Antler, Plating, 1973, 60, (5), 468

\title{
The Mode of Pore Closure in Electrodeposited Gold
}

In its early stages any electrodeposit is discontinuous, but as the crystal nuclei grow they rapidly form a coherent coating while the thickness is still quite small. While the great majority of the growing grains will coalesce perfectly a few will not, the gaps remaining as pores-a phenomenon only too well known to the electroplater and to the user of his deposits. At a sufficient thickness, of course, most deposits become pore-free by a process of lateral growth and closure of the pores.

An interesting approach to the mechanism by which pore closure occurs in gold electrodeposits-partly theoretical but also based upon extensive pore resistance measurements and porosity testing-has now been made by Dr M. Clarke of the City of London Polytechnic (Trans. Inst. Met. Fin., 1973, 51, (9), 150). Beginning with the premise that the small area of substrate at the bottom of a pore has some peculiarity that prevents nucleation and interferes with lateral growth-Dr Clarke calls these areas pore precursorshe goes on to examine the manner in which pores close. These precursors may be insulating inclusions or areas whose structure has been disturbed by rolling, scratching or polishing. Sealing can thus be accomplished only by the edges growing together with increasing thickness, leaving an enclosed cavity that is presumably filled with a minute amount of the electrolyte. No direct connection was observed between porosity and cathode efficiency.

Working with an almost neutral phosphate-buffered gold cyanide electrolyte free from alloying additions, Dr Clarke has shown that as the thickness of deposit increases the walls of a pore develop a shape with an increasingly steep slope similar to that of a volcano. Thus until a pore is sealed the area of exposed substrate is not significantly altered, although the pore opening at the deposit surface will have a much smaller area.

Despite a somewhat highly mathematical approach to the problem, the mechanism put forward by $\mathrm{Dr}$ Clarke should prompt the accumulation of further data on pore closure in a wider range of electrolytes and so promote the better practical utility of gold electrodeposits in industry. 